\title{
A CASE REPORT OF BLOUNT'S DISEASE IN PHYSIOTHERAPEUTIC PERSPECTIVE
}

\author{
Rajani Cartor Medidi *1, Mallireddy Raja Rajeswari ${ }^{2}$.
}

${ }^{*}$ Professor and Principal, VAPMS College Of Physiotherapy, Visakhapatnam, Andhra Pradesh, India. ${ }^{2}$ VAPMS College Of Physiotherapy, Visakhapatnam, Andhra Pradesh, India.

\section{ABSTRACT}

Background: This report on Blount's disease after evaluation and sessions of exercises has shown the difficulties of obese patient in performing exercises with post surgical weakness.

Case Summary: Blount Disease (Tibia Vara) Idiopathic tibia vara, or Blount disease, is the most common pathologic disorder producing a progressive genu varum deformity. It is characterized by abnormal growth of the medial aspect of the proximal tibial epiphysis, resulting in a progressive varus angulation below the knee. Tibia vara can occur at any age in a growing child. It is classified according to the age: infantile (1 to 3 years), juvenile ( 4 to 10 years), and adolescent (great than 11 years). The infantile group is the most common. The degree of deformity is measured by the distance between the two medial femoral condyles when the patient is lying. Multiple factors such as ethnicity, genetics, and mechanical stress are thought to be contributing elements to this disease. Diagnostic radiography is the method of choice in diagnosing Blount's disease in children, and common radiographic manifestations include a rounded, dome - like tibial metaphysis with fracturing or change in size of the epiphysis. During the earliest stage of infantile Blount disease, bracing has been utilized in an attempt to correct the varus angle of the proximal tibia.

Intervention: When performing osteotomies or with pin insertion, nerves as well as the anterior tibial artery and its recurrent branch, are at risk. With acute correction of the varus deformity, medial-side structures are at risk for stretch injury. Compartment syndrome is a risk particularly with acute correction of tibial deformity. Both through the surgical and/or conservative management, Physical therapy care should be continued to prevent muscle wasting, correct malalignment, strengthening the muscles, promote healing, weight reduction...etc

Conclusion: Obesity being one of the prime barrier to overcome and complete the exercises, use of aquatic therapy is favourable. Weakness of muscles have to be checked right from the time condition was diagnosed. Proper bracing and strengthening exercises along with moral support and gaining confidence are key for better prognosis.

KEY WORDS: Case Report, Blount's Disease, Congenital, Epiphyseal.

Address for correspondence: : Prof Dr Rajani Cartor Medidi MPT Ortho, MIAP, DHA, Principal, VAPMS College Of Physiotherapy, Visakhapatnam, Andhra Pradesh, India.

E-Mail: rajani.cartor@gmail.com

Access this Article online

Quick Response code

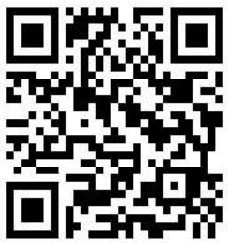

DOI: $10.16965 /$ ijpr.2019.155

Journal Information

International Journal of Physiotherapy and Research

ICV for 2016 ISSN (E) 2321-1822 | ISSN (P) 2321-8975

https://www.ijmhr.org/ijpr.html

DOI-Prefix: https://dx.doi.org/10.16965/ijpr

Article Information

Received: 16 May 2019

Peer Review: 16 May 2019

Revised: None
Accepted: $10 \mathrm{Jul} 2019$

Published (O): 19 Aug 2019

Published (P): 19 Aug 2019

\section{INTRODUCTION}

Blount's disease is a growth disorder of the tibia (shin bone) that causes the lower leg to angle inward, resembling a bowleg. This is an osteochondrodysplasia that results in inward turning of lower leg, located in tibia, which fails to develop normally. Blount disease is a familial infantile type defined as osteochondrosis deformans tibiae [1].

It is also known as Tibia Vara [2]. It is named after Walter Putnam Blount (1900-1992), an American pediatric orthopedic surgeon $[3,4]$. 
It has also been known as Mau-Nilsonne Syndrome, after C. Mau and H. Nilsonne, who published early case reports of the condition $[5,6]$.

Figure 1: Autosomal Recessive Inheritance - Blount's Disease. (Source: US national library of Medicine).

Autosomal recessive inheritance

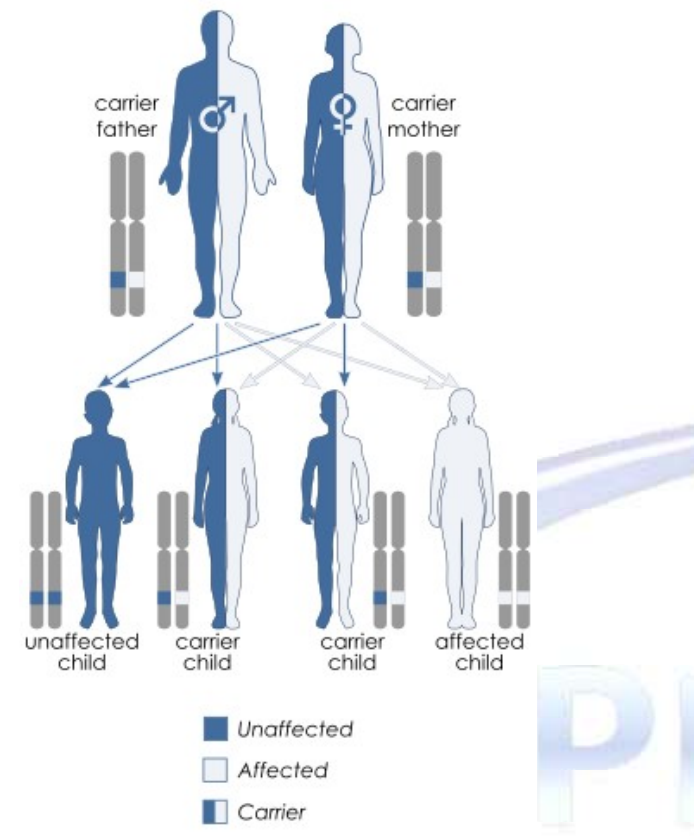

\section{Clinical Features}

Blount (1937) distinguished infantile and adolescent forms [1]. He described 22 cases of bowlegs in infants, with progressive deformity and radiologic findings of sloping proximal tibial epiphysis and a medial beak of the metaphysis. He suggested the existence of an infantile type with onset in the first year or two of life and an adolescent type developing just before puberty.

The description by Tobin (1957) of tibia vara beginning at puberty with osteochondritis dissecans of the knees in father and 2 sons suggested that the 2 disorders may be identical. Motzkin et al. (1992) and Tarnok et al. (2003) reported Blount disease in Bardet-Biedl syndrome [1].

Genu varum [also called bow-leggedness, bandiness, bandy-leg, and tibia vara], is a physical deformity marked by [outward] bowing of the lower leg in relation to the thigh, giving the appearance of an archers bow usually medial angulation of both femur and tibia is involved. Blount's disease is a deformity in the legs, mostly from the knees to the ankles. The affected bone curves in or out and forms the usual "archers bow" which can also be called bow-legs. There are two types of Blount's disease. The first type is infantile; this means that children under four years are diagnosed with this disease. Blount's disease in this age is very risky because sometimes it is not detected and it passes to the second type of Blount's disease.

The infantile form of Blount disease is common in the African Negro population of South Africa, although it is very rare in other groups. A series of 110 affected children, investigated in an attempt to elucidate the pathogenesis of this condition, demonstrates an approximately equal sex incidence while geographic distribution is patchy. Bowing of the legs, which is symmetrical in $82 \%$ is usually the presenting feature and develops at the onset of ambulation. There is no preceding illness, either local or systemic, and no dietary inadequacy. The children do not differ from their peers in age of walking, weight or range of joint movements. There are no biochemical or hematological abnormalities and no radiographic evidence of involvement of joints other than the knees. Twenty-two of the patients had never been carried in the traditional "picka-back" position and it is therefore unlikely that this posture is a significant causative factor. The children had a total of 231 siblings, of whom 10 had bowlegs, while 16 parents had been similarly affected during their own infancy. On this basis, Mendelian dominant or recessive inheritance is unlikely although it is possible that Blount disease is multifactorial in etiology [7]. A review of the English literature on Blount disease (osteochondrosis deformans tibiae; tibia vara) revealed that two forms of the disease, infantile and adolescent, are recognized. The cause of Blount disease is probably multifactorial. Most recent evidence on the pathogenesis implicates mechanical factors. The diagnosis can be difficult in very young children and must be based on history, physical examination, and radiographic findings. A proximal tibial metaphyseal-diaphyseal angle of greater than 11 degrees should be observed carefully for the development of Blount disease. Both nonoperative and operative treatment has been used successfully [8].

Genu varum is a relatively common finding in 
children. Physiologic bowing, which is seen most often, has a well-documented favorable natural history. Idiopathic tibia vara is the most common of the pathologic conditions that are associated with bowed legs; treatment strategies vary with the patient's age and the stage of disease and deformity. Genu varum may also accompany systemic conditions, such as achondroplasia, vitamin D-resistant rickets, renal osteodystrophy, and osteogenesis imperfecta-all of which can result in short stature. Indications for intervention are not always well defined. A rare disorder, focal fibrocartilaginous dysplasia, usually requires no treatment. Standing radiographs of the entire lower limbs are necessary for surgical planning, as the deformity can sometimes affect the distal femur rather than the proximal tibia. Restoration of the mechanical axis of the limb is the principal goal of treatment; the particular type of internal fixation is of secondary importance [9].

Blount disease is most likely to be caused by a combination of excessive compressive forces on the proximal medial metaphysis of the tibia and altered endochondral bone formation. It is unclear whether the deformity is caused by an intrinsic alteration of bone formation that is exacerbated by compressive forces or by compressive forces that cause a disruption in normal endochondral bone formation [10].

The combination of mechanical and biologic factors in tibia vara most likely influences the disease to varying extents. The mechanical forces contributing to the disease are the weight of the child, age at walking, and the varus deformity. In accordance with the heuter Volkmann principle compressive force across the medial femoral physis leads to growth retardation [11].

Damaged cartilage ossifies in a delayed fashion. As growth is selectively inhibited at the medial side of the knee due to these compressive forces result varus deformity progresses the posteriomedial aspect of the physis is most suppressed. Compressive forces across the medial femoral physis leads to growth retardation. The relationship between obesity and deformity is more linear in the late onset population than in early onset Blount disease. The result is a progressive angulation below the knee and an increase in the (compressive forces on the physis which changes the direction of the weight bearing forces on the upper tibial Epiphysis) form perpendicular to a oblique. The obliquity of this force tends to displace the tibial epiphyseal laterally. The addition to the delayed growth of the physis pressure on the adjacent Epiphysis leads to delayed ossification and intra-articular anomalies. Distal femoral valgus or varus deformity and or distal tibial varus or valgus. Deformities also can occur in conjunction with tibia vara. This deformity corrected at the same time of tibial vara deformity is corrected. Adolescent Blount Disease does not appear to be as progressive or as common as the infantile form [11].

\section{PATIENT INFORMATION}

Case Study: The first observance of slight deviation in the left leg was found to be noticed around $9^{\text {th }}$ month by the Child's parents. But now the child is Endomorphic (Obese) \& there was bilateral genu varum. Initially was under conservative knee bracing only. But later was advised for a surgery, upon which he has undergone, first surgery on $24^{\text {th }}$ august 2014 and second surgery on $24^{\text {th }}$ September 2014. Post surgery, those deviations which were an obvious problem had been corrected. Preoperatively he suffered bilateral knee pain and was unable to walk for a long periods. But post operation he was able to walk without pain and was then referred for physiotherapy. The child was using orthosis (KAFO) and dealing with his activities. Weight bearing exercises on the joints under obese conditions is quite a task challenging for the child and therapist. Instead aquatic therapy can unload the joints as well as motivating for the child in doing his exercises.

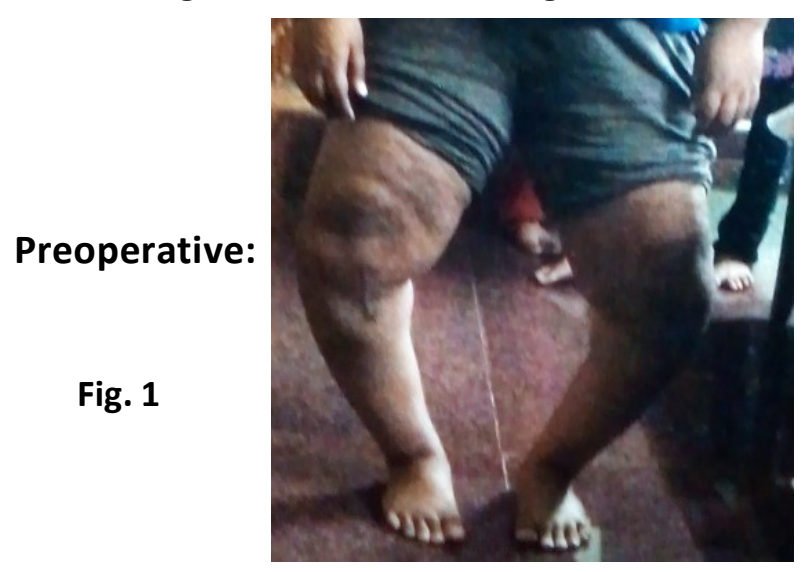


Bilateral Genu Varum (Anterior View):

Fig. 2

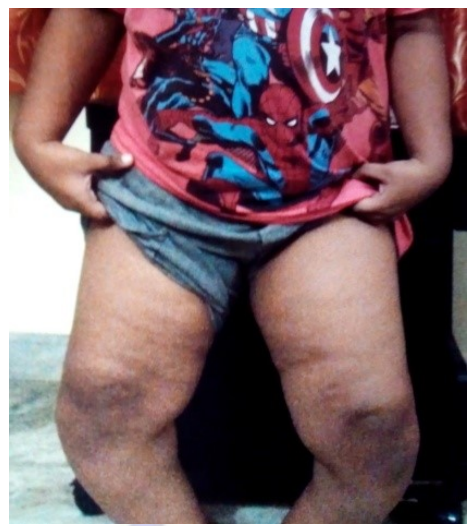

Bilateral Genu Varum (Posterior View):

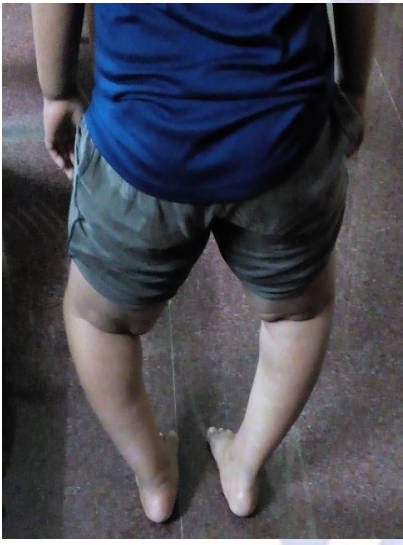

Fig. 3

Post Operative:

(Right operated)

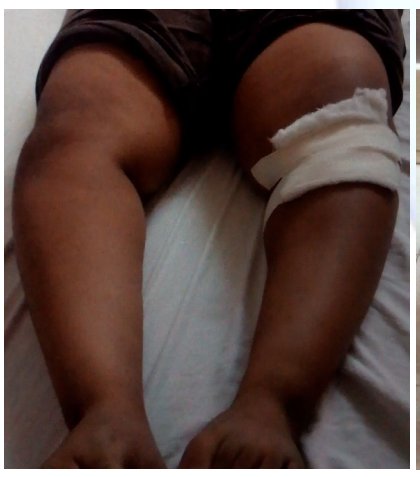

Fig. 5

In Orthosis

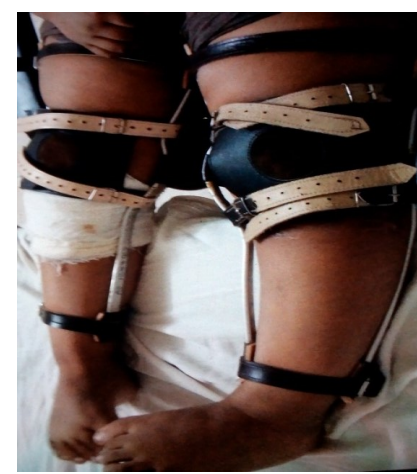

Fig. 7

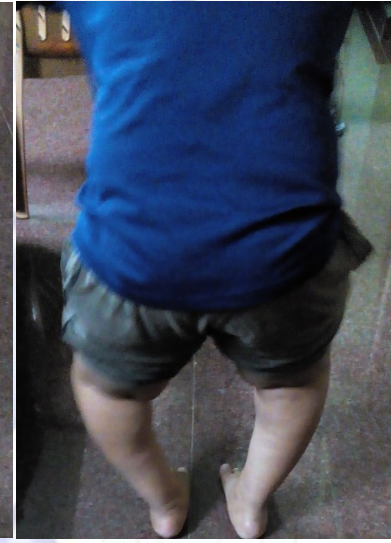

Fig.4

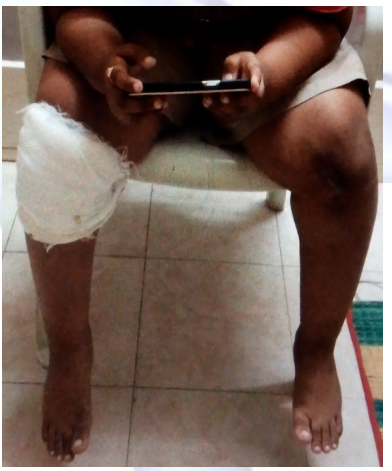

Fig. 6

After dressing removal

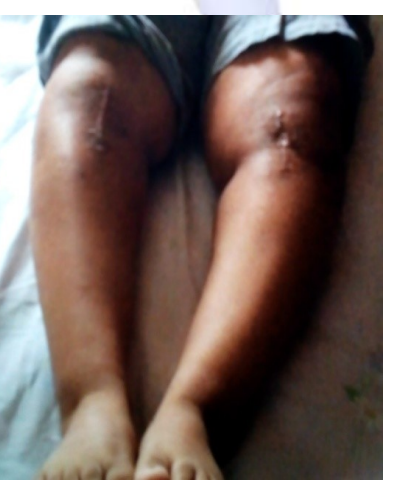

Fig. 8
In Crepe bandaging

Fig. 9

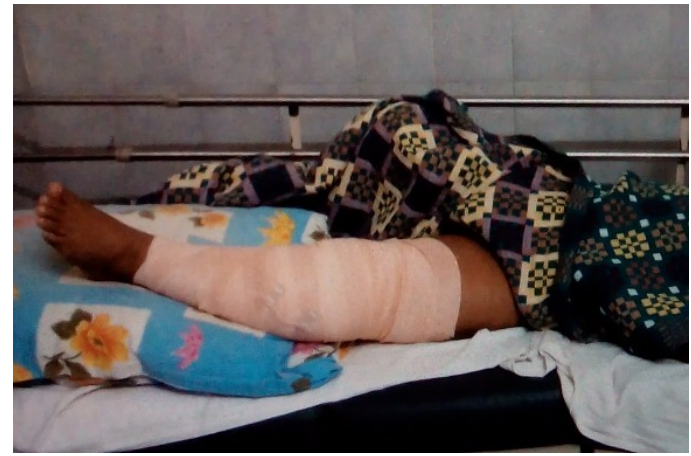

Fig. 10

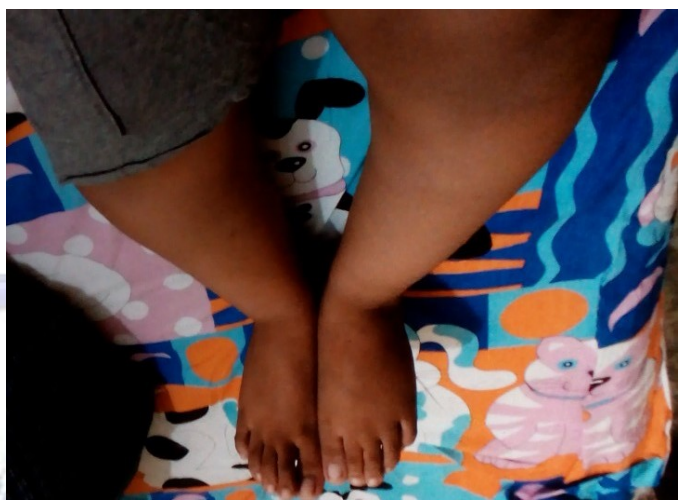

\section{ASSESSMENT}

Name

G. Ratan Lohith

Age

Gender

10 years

Address

Male

Madhurawada,

Visakhapatnam, Andhra Pradesh

Date of ASSESMENT : : 12-3-2016

\section{Chief Complaints}

: Difficulty in walking

Other Complaints : no relevant

Mode of Injury : : no relevant

Present History : varum knee

Past History : no relevant (knee pain)

Other Associated Problems : no relevant

Drug History : Vitamin - D Tablets

Family History : no relevant

Surgical History : $\quad 1^{\text {st }}$ surgery on 24-08-2014

$2^{\text {nd }}$ surgery in 26-09-2014

at BIRDD Hospital, Tirupathi

Personal History : no

Pain : Site-Knee

Side-Both Sides

Onset- with activity of lower limbs

Duration- continuous

Nature- Aching (crushing)

Relieving Factor - Rest

Aggrevative Factor - walk (weight bearing)

Intensity of pain - Mild 


\section{OBSERVATION}

Built

Attitude of Limbs

: Endomorphic

Posture [Standing]

Gait

External appliances orthosis

Skin Changes

Muscle Wasting
: bow legs

: Shoulder slightly forward Both Elbows semi flexed Knee semi flexed Foot eversion slightly : waddling gait Hip Knee ankle Foot

tibia vara is the physiologic genu varum deformity. It is difficult to differentiate radiographically between these two disorders in children younger than 2 years old [9].

Fig. 10: Radiograph showing the Genu varum.

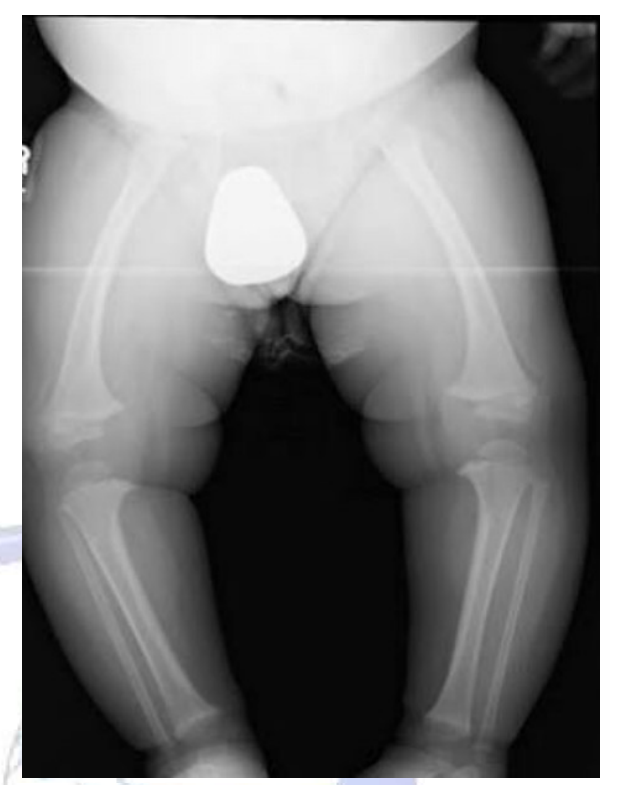

\section{INTERVENTIONS}

Generally no treatment is required for idiopathic presentation as it is a normal anatomical variant in young children. Treatment is indicated when persists beyond 3 and half years old, in the case of Unilateral presentation, or progressive worsening of the curvature.

Mild degree of deformity can be treated by wearing surgical shoes with $3 / 8$ outer raised and with a long inner rod extending to the groin and leather straps across the tibia and the knee. Corrective operations can also be performed, if necessary. The person would need to wear casts or braces following the operation. ${ }^{(8)}$ During childhood, assure the proper intake of vitamin $D$ to prevent rickets.

When the radiographic findings confirm the diagnosis, treatment should begin immediately. Orthotic management may be considered for children 3 years or younger with a mild deformity. Approximately $50 \%$ of children with this criterion may achieve adequate correction using orthoses.

Conservative management in the late-onset forms of tibia vara is contraindicated. The children are too large, compliance is poor and the remaining growth is too small to allow for adequate correction. 
The indications for surgical treatment in infantile tibia vara include 4 years of age or older, failure of orthotic management, and moderate to severe deformity. Proximal tibial valgus osteotomy with associated fibular diaphyseal osteotomy is the procedure of choice.

\section{MEDICAL TREATMENT}

Children who develop severe bowing before the age of 3 may be treated with knee ankle foot orthoses [2]. However, bracing may fail, or bowing may not be detected until the child is older. In some cases, surgery may be performed. ${ }^{\left({ }^{8}\right)}$ Surgery may involve cutting the shin bone (tibia) to realign it, and sometimes lengthen it as well. Other times, the growth of just the outer half of the tibia can be surgically restricted to allow the child's natural growth to reverse the bowing process. This second, much smaller surgery is most effective in children with less severe bowing and significant growth remaining.

Return to normal function and cosmetic appearance is expected if the knee can be properly aligned [12].

Conservative treatment can be an option in early onset Blount disease and consists of brace treatment. Brace therapy should be attempted in all children younger than 2.5 years with stage I or II disease. Ambulatory bracing with an abovethe -knee orthosis has been shown to prevent progression of disease. Bracing has been shown to correct both varus deformity and the pathologic proximal-medial tibial growth disturbance. If the disease continues to progress to stage III with brace treatment, it will no longer be effective. Other risk factors for failure of brace treatment include obesity; varus thrust age older than 3 years at initial treatment and bilateral disease [11].

\section{NON SURGICAL TREATMENT}

Non-operative treatment is an option in a select group of infantile Blount disease patients. If they are diagnosed before age 4 years, knee-ankle-foot-orthoses have a role in a Langenskiold stage I or II disease, especially with unilateral involvement[10]. Patient characteristics that predispose to failure of conservative treatment include varus thrust, age older than 3 years, weight greater than $90^{\text {th }}$ percentile, bilateral disease and Langenskiold grade higher than 3.

In late-onset patients and early onset patients in whom brace management fails, operative intervention is indicated for increasing severity of symptoms or progression of deformity

Surgical intervention is contraindicated in children younger than 2 years because it is difficult at this age to differentiate between Blount disease and excessive physiologic bowing that may resolve spontaneously.

A knee ankle foot orthosis (KAFO) is a common method of bracing. This type of support fixes the knee in extension and contains a medial bar that applies valgus pressure to the joint. The medial joint is then relieved of excess pressure and normal growth along the epiphysis will hopefully resume. For best result it is recommended that the brace be worn 23 hours a day for approximately 2 years depending on the degree of varus angulation [6].

The actual effectiveness of the method is controversial however bracing is not successfull for every patient. Severe obesity, bilateral deformity and age greater than 3 years are the factors found to increase [11].

The rate of brace failure additionally with a lack of scientific evidence resulted in disregard by many physicians [12].

This method a retrospective observation by shinohara et al discovered that in a study of 46 limbs left untreated for Blount disease I -III 40 limbs resolved spontaneously. Recovery time was also found to be no sooner than a patient using braced $[6,11]$.

\section{SURGICAL TREATMENT}

The incidence of infantile Blount disease is rising in parallel to the increasing obesity in children.

This disease should be differentiated from physiological genu varum, and the potential psychosocial and physical complications are prevented with early diagnosis and treatment [12].

The obesity associated with Blount disease leads to additional risk factors for surgery owing to their obesity patients are at higher risk for obstructive sleep apnea and should be screened during their preoperative workup in 
order to adequately treat these patients postoperatively [13].

Twelve patients, seventeen to twenty-five years old, who had had infantile Blount's disease involving nineteen limbs, were evaluated at a mean of twelve years after an initial osteotomy. Twelve of the nineteen knees were symptomatic, and eight of the symptomatic knees showed early degenerative changes by arthroscopy or arthrotomy. There was a direct correlation between the symptoms and the severity of the involvement of the proximal end of the tibia. A poor final outcome appears to be related to physeal damage. Early osteotomy must be performed before permanent physeal damage and subsequent incongruity of the joint occur [14].

Surgery is often recommended in patient who have not responded to brace treatment, children who have severe deformities and in children who are older and are no longer candidates for bracing. Several surgeries are available to treat Blount disease, including osteotomies hemiepiphysiodesis.

An Osteotomy is a procedure that involves cutting and realigning bone to put it in a more normal position this type of surgery usually corrects the deformity immediately

A hemiepiphysiodesis on the other hand corrects the deformity over time and involves placing plates or staples over the growth plate on one side of the tibia and or femur bone to stop the growth on that side. The other side then continues to grow while the plate guides the growth of an already angulated bone into a straighter position over time [15].

Performing total knee arthroplasty in patients with Blount disease or Blount like deformity despite a challenging patient population in which to perform total knee arthroplasty, we show that it can be done with a low risk of complication.

Seventeen patients with infantile Blount disease who were treated by surgical correction and followed up to skeletal maturity were reviewed by clinical and radiographic examination. Patients with symptomatic knees or significant ligament instability or both underwent further evaluation by magnetic resonance imaging (MRI) or arthroscopy or both. Average age at time of initial surgery was 5.5 years and at final follow-up was 20.5 years. Average length of follow-up was 15 years. Recurrence of the deformity requiring repeated osteotomy occurred more frequently in children who underwent initial osteotomy at $>4$ years of age or at Langenskiöld stage $>$ or $=$ III or both. Patients who underwent a single osteotomy for correction of their deformity had significantly decreased pain in the affected knee at maturity. All patients who were symptomatic or had significant knee instability or both had abnormal ligamentous, meniscal, or bony changes (or more than one of these) on MRI, which were confirmed by arthroscopy. Early surgical intervention during initial stages of the disease process will result in a decreased incidence of recurrence of deformity and decreased symptoms and knee pathology at skeletal maturity [16].

The overcorrection group had non-statistically significant recurrence compared to the nonovercorrection group, and overcorrection more than valgus $15^{\circ}$ has no benefit to prevent recurrence [17].

\section{INFANTILE BLOUNT DISEASE}

Surgical intervention is indicated in early-onset patients who develop Blount disease changes consistent with Langenskiold stage III or IV Bracing has not been shown to be effective past these stages, and the disease will continue to progress. Surgical options include corrective proximal tibial osteotomies with various fixation methods used sometimes in concert with guided growth or hemiepiphysiodesis [11].

\section{ADOLESCENT BLOUNT DISEASE}

In individuals with adolescent tibia vara, observation is indicated only with painless, non-progressive, mild deformities. In contrast to infantile Blount disease, which can be corrected in the earlier stages with bracing, the adolescent form of the disease has not been shown to respond to Non operative treatment. Surgical treatment depends on the stage of the disease and the skeletal age of the child. As with the infantile form of the disease, there are multiple surgical approaches to correcting, which include proximal tibial osteotomy [17].

A drawback of this technique is the unpredict- 
able growths from the diseased medial tibial physis and therefore unpredictable correction. This technique has been show to be less effective in obese patients and more severe deformity.

Growth with compressive plating across the convexity of the growth plate is an option in those with significant growth remaining. Guided growth can also be used concomitantly to correct less severe deformity of the distal femur while correcting the proximal tibia however both guided growth and hemie pi-physiodesis fail to address sagittal plane deformity or limb length discrepancy [17].

\section{POSTOPERATIVE CARE}

In the postoperative period, it is important to carefully monitor the neurovascular status because patients who undergo corrective proximal tibial osteotomies are at risk for compartment syndrome. Compartment syndrome must be recognized and treated early. Owing to the increased incidence of obesity, patients with Blount disease are at significant risk for deep venous thrombosis, especially in the adolescent population, and patients therefore should be onchemoprophylaxis postoperatively. In an effort to determine whether bracing is effective in reversing early infantile Blount's disease, 27 patients with Langenskiold stage II disease were studied. Ten patients had bilateral disease (two of these patients had stage III disease affecting one side). Age at brace initiation averaged 2.1 years (range, 1.5-3.2). The duration of bracing averaged 9.7 months. Follow-up averaged 5.9 years. Successful outcomes (improved alignment without the need for osteotomy) were achieved in 19 (70\%) patients, the majority of them having unilateral disease. Of the 37 affected extremities, 24 (65\%) had successful outcomes. Eight patients (13 extremities) required tibial osteotomies and were classified as bracing failures. Of the 10 patients with bilateral involvement, seven (70\%) required osteotomies for one or both extremities, whereas only one (6\%) of 17 patients with unilateral involvement required osteotomy. Bracing appears to be effective in stage II infantile Blount's disease, particularly in those with unilateral involvement. Children with bilateral disease are most at risk for requiring subsequent corrective osteotomy [19].

\section{ROLE OF PHYSIOTHERAPY}

Gradual Knee mobilization was the main part of the treatment given. Knee mobilization has been initiated in a small range relaxed as free movement progressing gradually. Thermotherapy (heat modalities) adjunct was used as a pain controlling measure. Strengthening Exercises for quadriceps, hamstrings and gluteus muscles were given. When the child started to walk, he was given correct training for standing, balancing, weight transferring and walking. Correct method of applying orthosis, weight transfers, and proper ambulation have been taught for few days. A knee ankle foot orthosis (KAFO) is a common method of bracing [18].

As this type of support fixes the knee in extension and contains a medial bar that applies valgus pressure to the joint. The medial joint is then relieved of excess pressure and normal growth along the epiphysis will hopefully resume. For best result it is recommended that the brace be worn 23 hours a day for approximately 2 years depending on the degree of varus angulation. When the child was allowed to walk,weighttransfer was allowed gradually in a progressive manner till an independent ambulation was achieved. Of course, a keen observation was given to find out any recurrences arising.

\section{FOLLOW UP \& OUTCOMES}

Exercises have maintained the muscle and thus also in proper postural maintenance. As the condition and the age of the patient with skeletal embryonic development still on its way to reach maturity, child is bound to undergo revision of surgical procedures for the prevailing changes in the joint. Follow up has shown the patient's suffering go into submission post surgical but sooner coming back. Out of all, the increment in the weight along with inability to do exercises has setback the patient.

\section{DISCUSSION}

As weight being one of the prime hindrances in the sustenance of exercises, the treatment chosen is hydrotherapy in a therapeutic pool, where the joints will be out of weight. This helps in performing the exercises without pain. Thus patient feels interested and confident in doing 
those exercises.

Patient Perspective: Patient (child) feels better after certain set of exercises given for a period of time. His interest levels have increased with pool therapy. His dependency has decreased and that led to his active participation in various streams of life.

\section{Conflicts of interest: None}

\section{REFERENCES}

[1]. OMIM Entry - 259200 - BLOUNT DISEASE, ADOLESCENT". omim.org. Retrieved 7 November 2017.

[2]. Clarke SE, McCarthy JJ, Davidson RS. Treatment of Blount disease: a comparison between the multiaxial correction system and other external fixators. J Pediatr Orthop. 2009; 29(2): 103-9. doi:10.1097/BPO.0b013e3181982a62. PMID 19352232.

[3]. synd/1470 at Who Named It?

[4]. W. P. Blount. Tibia vara: osteochondrosis deformans tibiae. Journal of Bone and Joint Surgery, Boston, 1937;19: 1-29.

[5]. Mau, C. Genu varum bedingt durch Tihiaepi physendefekt bei Kartilaginärer Exostose. Z. orthop. Chir. 1923;24(44):383.

[6]. Nilsonne, H. Genu varum mit eigentümlichen Epiphysenveränderungen. Acta chir. scand. 1929;44:187.

[7]. Bathfield ca,Beighton blount disease.a review of etiological factors in 110patient clin orthop relat res. 1978;135:29-80.

[8]. Bradway Jk, Klassen Ra. Peterson ha.blount disease:a review of the english literature.j pediatric orthopedics.1987jul-aug;7(4):472-80.

[9]. Brooks Wc, Gross Rh. Genu varum in children diagnosis and treatment. J am acad orthop surg.1995 nov;3(6):326-335.
[10].Sanjeev Sabharwal, J Bone Joint Surg Am. 2009;91:1758-1776.doi:10.2106/JBJS.H.01348,

[11]. Davids Jr, Huskamp M, Bagley Am. A dynamic biomechanical analysis of the etiology of adolescent tibia vara .j pediatr orthop 1996 jul- aug;16(4):4618.

[12]. Orthobullets infantile blount disease tibia vara. aug 2014;30. http; //www.orthbullets .com/pediatrics/4050/infantile-blount disease.

[13]. Ayla Güven, MD, Suna Hancýlý, MD, L Ýhsan Kuru, Obesity and Increasing Rate of Infantile Blount Disease, clin pediatr.2014jan 13;53 (6) :539-543.

[14]. Gordon je Hughes ms, Shephered k, szymanski da schoenecker pl,parker I. obstruction sleepapnoea syndrome in morbidly obese children with tibia vara. j bone joint surg br.2006jan;88(1):100-3.

[15]. Hofmann a, Jones re Herring ja blounts disease after skeletal maturity. j bone joint surg am.1982 sep;64 (7):1004-9.

[16]. McCarthy JJ, Maclntyre NR, Hooks B, Davidson RS. Double osteotomy for the treatment of severe Blount disease. J Pediatr Orthop. 2009;29 (2):1159. doi:10.1097/BPO.0b013e3181982512. PMID 19352234.

[17]. Doyle bs, volk ag Smith; infantile blount disease :long -term follow -up of surgically treatment patients at skeletal maturity j pediatr orthop 1996 jul aug;16(4)469-76.

[18]. Eamsobhana p, Kaewpornsawan k, Yusuwank. Do we need to do overcorrection in blount disease int orthop 2014;8(38):1661-4.

[19]. Alsancak, S; Guner, S; Kinik. Orthotic variations in the management of infantile tibia vara and the results of treatment. Prosthetics and Orthotics International. 2013 ; 37 (5): 375-83. doi:10.1177/ 0309364612471369 . PMID 23344116.

[20]. Richards bs, katz de Sims jb. Effectiveness of brace treatment in early infantile blount disease. J Pediatr Orthop 1998 may -june;18(3):374-80.

How to cite this article: Rajani Cartor Medidi, Mallireddy Raja

Rajeswari. A CASE REPORT OF BLOUNT'S DISEASE IN PHYSIOTHERAPEUTIC PERSPECTIVE. Int J Physiother Res 2019;7(4):3188-3196. DOI: 10.16965/ijpr.2019.155 